\title{
Comparison between Conventional Radiography and High Resolution Computed Tomography in Interstitial Disease of Lungs
}

\author{
P. Madhu ${ }^{\oplus 1}$, D. Santhosh ${ }^{\odot 2}$ \\ ${ }^{1}$ Associate Professor, Department of Radiodiagnosis, Government Medical College, Nizamabad, Telangana, India, ${ }^{2}$ Assistant Professor, Department of Radiodiagnosis, \\ Government Medical College, Nizamabad, Telangana, India.
}

\section{Abstract}

Background : Interstitial lung diseases are a diverse group of over 200 disease entities in which the primary site of injury is the lung interstitium. These diseases vary widely in their etiology, clinicoradiologic presentation, histopathological features and clinical course. HRCT has its limitations in the detection of interstitial lung diseases, its sensitivity is not $100 \%$ and the limitations of sensitivity are not well established. This study attempted to compare between conventional radiography and HRCT in the diagnosis of interstitial lung diseases. Subjects and Methods: All the patients were subjected to both conventional radiography and HRCT examinations and the images were viewed and analyzed. The two modalities were compared with regards to their ability to detect findings like nodular opacities, reticular opacities, septal thickening, honeycombing, lymphadenopathy etc. Results: The study has gender distribution of $60 \%$ males and $40 \%$ females. The main result of the study was that higher number of samples with findings were detected by HRCT than conventional radiography. In the detection of nodular opacity and septal thickening, the $\mathrm{p}$ value was less than 0.05 meaning the difference was statistically significant. Of the 30 patients, 2 had normal chest radiograms while HRCT was able to detect reticular opacities in these patients. The most common abnormality seen in both chest radiograms and HRCT was reticular opacities. The distinction between air space nodules and interstitial nodules was also much better appreciated on HRCT than on conventional radiography. In the detection of honeycombing, HRCT was much more specific being able to detect it in cases when the chest radiogram showed only reticular opacities. Even in the detection of other findings like air trapping and lymphadenopathy, HRCT scored over conventional radiography. Conclusion: HRCT therefore seems to be the investigation of choice in evaluating patients of interstitial lung disease.

Keywords: Conventional Radiography, High Resolution Computed Tomography, Interstitial Disease of Lungs.

Corresponding Author: D. Santhosh, Assistant Professor, Department of Radiodiagnosis, Government Medical College, Nizamabad, Telangana, India.

E-mail: drsanthosh145@gmail.com

Received: 27 December 2019

\section{Introduction}

Interstitial lung diseases are a diverse group of diseases which affect the lung interstitium and share similar clinical and radiological manifestations. They are a heterogeneous group of disorders of the lower respiratory tract that are characterised by both acute and chronic inflammation and a generally irreversible and relentless process of fibrosis in the interstitium and the alveolar walls. ${ }^{[1]}$ The interstitium refers to tissues of the alveolar wall between the capillary endothelium and the alveolar epithelium and it is the site of primary injury. The term "interstitial" can be misleading as most of these conditions also affect the airway spaces and even the blood vessels, but it is the predominant and primary involvement of the interstitium that characterizes them. The interstitial lung diseases are a heterogenous group of many acute and chronic pulmonary disorders. Though individually rare, as a group, they are a common clinical problem. Though they are grouped together, there are great variations in the risk factors for their development, their pathological processes, the relevant therapies and the associated prognosis, making an accurate diagnosis very essential. ${ }^{[2]}$

For the physician, the distinctive sign of interstitial lung disease is the evidence of diffuse pulmonary opacities on chest radiograph or a suggestive pattern on pulmonary function tests. The diagnosis of chronic ILD depends on epidemiologic data, clinical and radiological findings which make it possible to consider a diagnosis of high probability in at least $60 \%$ of cases and reduce the gamut of hypothesis in the remaining.

In the diagnosis of interstitial lung diseases, clinical, radiological and histological correlation is needed on most occasions. 
The chest radiograph remains the basic radiological tool in the investigation of these patients. However, chest radiograph is relatively insensitive and is normal in $10-20 \%$ of patients with histologically proven interstitial lung disease. Many diseases remain occult or are not correctly diagnosed on chest radiograph, appearing as a non specific 'reticulonodular pattern. It is not specific also in that different interstitial lung diseases can have similar radiographic appearances.

High-resolution computed tomography of the chest has become an invaluable tool in the diagnostic process of interstitial lung diseases. A confident diagnosis can often bemade on the basis of high-resolution computed tomographic findings and the clinical context. Serologic testing can be helpful in selected cases. Improvements in CT scanner technology has now made it possible to image the lung parenchyma with excellent anatomic detail. The morphologic characteristics of diffuse parenchymal lung diseases can be demonstrated with very high resolution. HRCT or high resolution computed tomography is more sensitive than conventional chest radiography in the detection of interstitial lung diseases. However, sensitivity is not $100 \% .{ }^{[3]}$ The specificity for the characterization of different lung diseases has been documented and appears to be better than conventional radiography. The ability to characterize different disease processes and to provide a specific diagnosis by HRCT is a big advantage in clinical situations.

To date, numerous reports have documented that HRCT is more sensitive and specific than chest radiography in establishing a diagnosis in diffuse lung diseases. HRCT has proved particularly accurate in establishing the diagnosis of silicosis, idiopathic pulmonary fibrosis, lymphangitic carcinomatosis, and sarcoidosis. In general, the accuracy of chest radiograph film diagnosis in the same disorders was much lower.

Three different observers independently interpreted plain chest radiograph films and HRCT scans, listed the three most likely diagnoses, and assigned a degree of confidence for the first- choice diagnosis. The highest confidence level was reached with $49 \%$ of CT scans and $23 \%$ of plain chest films, and a correct diagnosis was made with $93 \%$ and $77 \%$, respectively. Based on clinical information alone, a confident correct diagnosis could be made in $29 \%$ of the cases. Combined interpretation of clinical data and chest radiograph film findings increased the confidence in a correct diagnosis to $54 \%$, and to $80 \%$ when clinical, radiographic, and HRCT findings were analyzed together. Consequently, most patients with a diagnosis of diffuse lung disease based on plain films will proceed to HRCT to narrow down the differential diagnosis or even to establish a specific diagnosis and to do so with a higher confidence level. ${ }^{[4]}$ Moreover, although there have been numerous studies comparing conventional radiography and HRCT in the diagnosis of specific interstitial diseases, very few studies have incorporated the whole gamut of interstitial lung diseases in a single study.

\section{Subjects and Methods}

A cross sectional study was performed. All ages and both sexes were included in the study. It was a duration based study. 30 patients were included in the study. All the patients with clinical suspicion of interstitial lung disease who were referred to the department of Radiodiagnosis, for diagnosis and evaluation were subjected to both conventional chest radiograph and HRCT. Diagnosis was based on clinical and radiographic findings.

\section{Inclusion criteria}

Patients presenting with collagen vascular diseases like systemic lupus erythematosus, rheumatoid disease, systemic sclerosis, systemic vasculitides like Wegener's granulomatosis, pulmonary tuberculosis with disseminated disease status, industrial exposure related diseases like asbestosis, silicosis, coal worker's pneumoconiosis, etc

medication, drugs and radiation exposure related cases of idiopathic interstitial pneumonias and hypersensitivity

pneumonias ,cases of allergic bronchopulmonary aspergillosis, invasive aspergillosis and lymphangitic spread of tumours.

\section{Duration of the study}

The study was conducted for a period of 18 months.

HRCT scans were performed in supine position in a Siemens 16 Slice CT machine in suspended inspiration using a $\mathrm{kVp}$ of 130 and $\mathrm{mAs}$ of $60-70$. The window width was set between $1200-1500$ and the window level at -600 to -700 . The matrix used was $512 \times 512$ while the pitch was set at 1:1.

The patients also underwent chest radiography posteroanterior view at $60 \mathrm{kVp}$ and 5-8 mAs in a SIEMENS $500 \mathrm{~mA}$ xray machine.

\section{Results}

The 30 patients were subjected to both conventional chest radiograph and HRCT scan thorax and a detailed work up of these patients was performed; their clinical history, relevant past and occupational history and any laboratory data recorded.

Of the 30 patients, 18 patients were males (60\%) and $12(40 \%)$ were females. The age of the patients ranged from 24 years to 74 years.

Sarcoidosis (23.3\%), systemic lupus erythematosus (10\%), rheumatoid arthritis $(10 \%)$, idiopathic pulmonary fibrosis (13.3\%), silicosis (16.7\%), disseminated tuberculosis $(6.7 \%)$, 


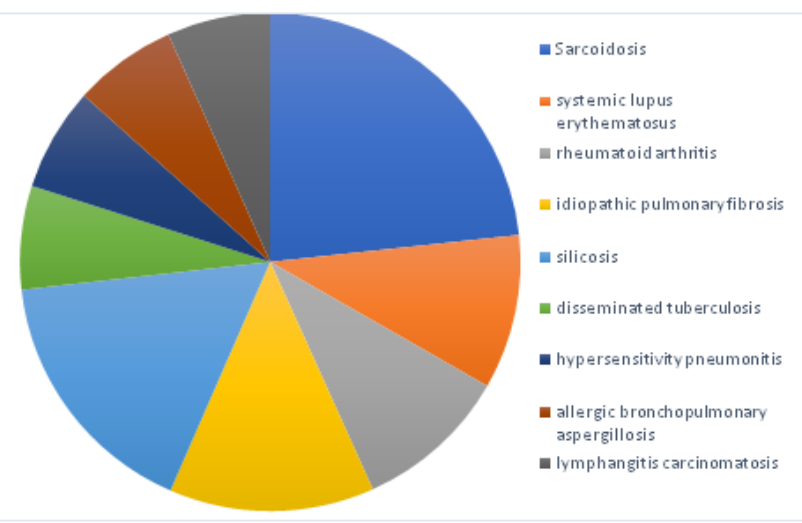

Figure 1: The comparative tables between chest radiograph and HRCT in the detection of different findings

hypersensitivity pneumonitis (6.7\%), allergic bronchopulmonary aspergillosis $(6.7 \%)$ and lymphangitis carcinomatosis $(6.7 \%)$.

Higher no. of samples with reticular opacity and nodular opacity were detected in HRCT method compared to Radiograph method but the difference between the two methods was not statistically significant $(\mathrm{P}>0.05)$.

The results of our investigation were evaluated using proportions and chi squared test. The level of significance was 0.05 . We compare the P-Value with the level of significance. If $\mathrm{P}<0.05$, we reject the null hypothesis and accept the alternate hypothesis. If $\mathrm{P} \geq 0.05$, we accept the null hypothesis.

\section{Discussion}

The main observation in our study was that higher number of samples with findings were detected by HRCT as compared to conventional radiography. Even when both modalities were able to detect the findings, HRCT could characterize the abnormality and specify its location much more accurately. The chest radiograph can appear completely normal in patients suffering from interstitial lung diseases. Therein lays the inherent lack of sensitivity of conventional chest radiograph in the diagnosis of the conditions. In our study, 2 of the 30 patients $(6.7 \%)$ had no abnormalities in their chest radiographs. However HRCT was able to show reticular changes in these patients.

Higher no. of samples with reticular opacity were detected in HRCT method compared to chest radiograph method but the difference between the two methods was not statistically significant $(\mathrm{P}>0.05)$. The most common abnormality seen on chest radiographs was reticular opacities which were observed in $73 \%$ of the cases. However HRCT managed to detect reticular opacities in $90 \%$ of the cases, thereby
REPRESENTATIVE CASES

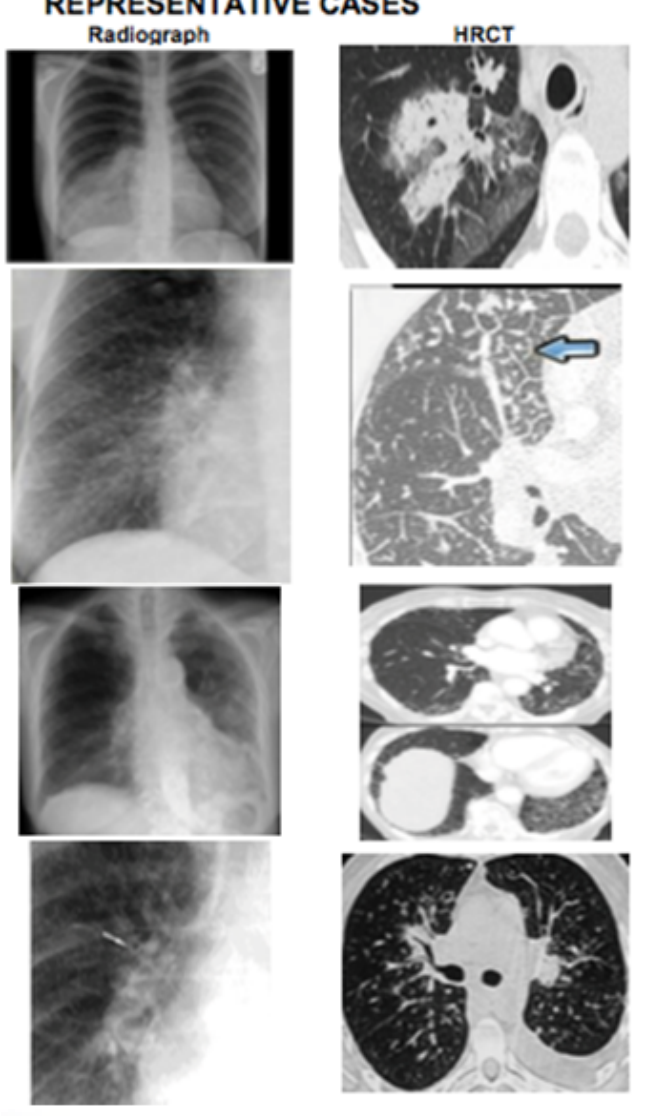

Figure 2: Representative Cases.

implying a much greater sensitivity in the identification of these densities. Furthermore, in the detection of these reticular opacities, although conventional chest radiography was able to differentiate between medium and coarse opacities, their detection of fine reticular densities was a cause of concern. HRCT detected fine reticular opacities in the lungs when the chest radiograph revealed no such abnormalities.

Higher no. of samples with nodular opacity were detected in HRCT method compared to chest radiography method and this difference between the two methods was found to be statistically significant $(\mathrm{P}<0.05)$. Nodular opacities are another very common manifestation of interstitial lung diseases. In our study $37 \%$ had nodular opacities in their chest radiographs. While HRCT showed evidence of nodular opacities in 63\% of the cases. The appearance of the nodules themselves can be an indicator as to whether they are interstitial or air space nodules. Interstitial nodules tend to be sharply marginated while air space nodules poorly defined. This distinction of nodules is much better appreciated on HRCT scans than on chest radiograms. 


\begin{tabular}{|c|c|c|c|c|c|c|c|}
\hline \multirow[t]{2}{*}{ Reticular } & \multicolumn{2}{|c|}{ HRCT } & \multicolumn{2}{|c|}{ Radiograph } & \multirow[t]{2}{*}{ Total } & \multirow[t]{2}{*}{$\chi^{2}$} & \multirow[t]{2}{*}{ Total } \\
\hline & $\mathrm{n}$ & $\%$ & $\mathrm{n}$ & $\%$ & & & \\
\hline Present & 27 & 90 & 22 & $73 \%$ & 49 & 2.783 & 0.095 \\
\hline Absent & 3 & 10 & 8 & $27 \%$ & 11 & & \\
\hline \multicolumn{8}{|l|}{$\begin{array}{l}\text { Nodular } \\
\text { Opacity }\end{array}$} \\
\hline Present & 19 & 63 & 11 & $37 \%$ & 30 & 4.267 & $0.039 *$ \\
\hline Absent & 11 & 37 & 19 & $63 \%$ & 30 & & \\
\hline \multicolumn{8}{|c|}{$\begin{array}{l}\text { Septal thick- } \\
\text { ening }\end{array}$} \\
\hline Present & 15 & 50 & 6 & $20 \%$ & 21 & 5.934 & 0.015 \\
\hline Absent & 15 & 50 & 24 & $80 \%$ & 39 & & \\
\hline \multicolumn{8}{|c|}{ Honeycombin } \\
\hline Present & 10 & 33 & 6 & $20 \%$ & 16 & 1.364 & 0.243 \\
\hline Absent & 20 & 67 & 24 & $80 \%$ & 44 & & \\
\hline \multicolumn{8}{|c|}{$\begin{array}{l}\text { Traction } \\
\text { bronchiecta- } \\
\text { sis }\end{array}$} \\
\hline Present & 12 & 4 & 9 & $30 \%$ & 21 & 0.659 & 0.417 \\
\hline Absent & 18 & 60 & 21 & $70 \%$ & 39 & & \\
\hline \multicolumn{8}{|c|}{ Consolidation } \\
\hline Present & 13 & 43 & 13 & $43 \%$ & 26 & & \\
\hline Absent & 17 & 57 & 17 & $57 \%$ & 34 & & \\
\hline \multicolumn{8}{|c|}{$\begin{array}{l}\text { Ground } \\
\text { glassopacity }\end{array}$} \\
\hline Present & 13 & 43 & 10 & $33 \%$ & 23 & 0.635 & 0426 \\
\hline Absent & 17 & 57 & 20 & $67 \%$ & 37 & & \\
\hline \multicolumn{8}{|c|}{ Lymphadenor } \\
\hline Present & 16 & 53 & 10 & $33 \%$ & 26 & 2.443 & 0.118 \\
\hline Absent & 14 & 47 & 20 & $67 \%$ & 34 & & \\
\hline
\end{tabular}

*denotes significant association

Furthermore, the location and distribution of nodules in relation to lung structures is a key determinant in narrowing down the differential diagnosis. Nodules can be classified as perilympahtic, random and centrilobular based on their distribution on HRCT. Perilympahtic nodules occur in relation to lung lymphatics and in clinical practice is usually the result of sarcoidosis. In our study this perilymphatic distribution of nodules in sarcoidosis was well appreciated. Similarly, random nodules are most typical of military tuberculosis, fungal infections or hematogenous metastases. Such narrowing down of the differential diagnosis based on the nodule distribution was possible only on HRCT and not on chest radiography. The maximum that chest radiography could offer in this context was to show a subpleural distribution of the nodule.
This highlights the increased specificity of HRCT over conventional radiography.

Higher no. of samples with honeycombing were detected in HRCT method compared to X-ray method but the difference between the two methods was not statistically significant $(\mathrm{P}>0.05)$. The end stage of interstitial lung disease is characterized by honeycombing. It reflects extensive lung fibrosis with alveolar destruction, thereby resulting in a characteristic reticular appearance. ${ }^{[5]}$ On HRCT, it is associated with gross distortion of lung architecture, where individual lobules are no longer visible. In our study, such honeycombing was seen in $33 \%$ of the cases on HRCT while chest radiography could detect them in only $20 \%$. On HRCT, honeycombing was much more accurately diagnosed by the 


\begin{tabular}{|lll}
\hline Table 2: Comparative Findings in Radiograph and HRCT & & \\
\hline Variables & Radiograph & HRCT \\
\hline Air space consolidation & 14 & 19 \\
\hline Septal thickening & 12 & 19 \\
\hline Reticular opacities & 13 & 29 \\
\hline Reticular opacities - Medium & 11 & 16 \\
\hline Reticular opacities - Coarse & 2 & 6 \\
\hline Peribronchial cuffing & 7 & 13 \\
\hline Honeycombing & 4 & 6 \\
\hline Nodular opacities & 13 & 18 \\
\hline Distribution of nodules(perilymphatic, centrilobular or random & NA & A \\
Ground glass opacity & 5 & 12 \\
\hline Traction bronchiectasis & 4 & 11 \\
\hline Air trapping & 2 & 6 \\
\hline Hilar and mediastinal lymphadenopathy & 6 & 16 \\
\hline
\end{tabular}

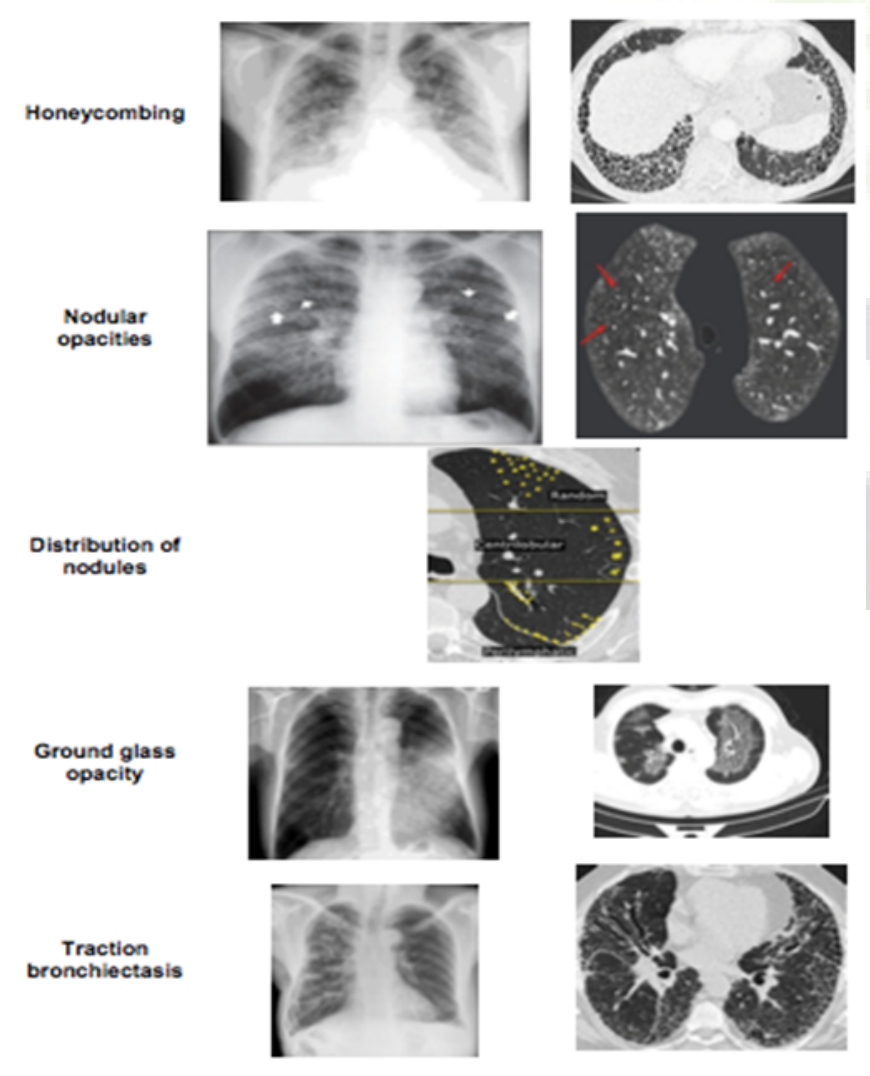

Figure 3: Patterns on X Ray.

presence of thick walled, air filled cysts, usually measuring $3 \mathrm{~mm}$ to $1 \mathrm{~cm}$ in diameter, typically occurring in several layers at the pleural surface. Detection of honeycombing has great clinical significance as its presence strongly suggest the diagnosis of usual interstitial pneumonia. It also indicates end stage disease, whereby the patient will gain little from a lung biopsy and hence avoid it. In this context also, HRCT definitely scores over conventional radiography.

Higher no. of samples with traction bronchiectasis were detected in HRCT method compared to X-ray method but the difference between the two methods was not statistically significant $(\mathrm{P}>0.05)$. Traction bronchiectasis or bronchial dilatation resulting from lung fibrosis was visible in $30 \%$ of the cases on chest radiography. They were typically associated with reticular opacities and in some cases with honeycombing. HRCT however managed to detect traction bronchiectasis in $40 \%$. The bronchiectasis in these cases was typically associated with an absence of mucous plugging or fluid within the bronchi. This finding or the absence of it was much better appreciated on HRCT than conventional radiography.

The detection of associated air trapping and lymphadenopathy was also greater with HRCT than with conventional radiography. HRCT abnormalities, but not the chest radiographic stage, were strongly associated with functional parameters. Abnormal changes of lung parenchyma, established by HRCT features, were associated with respiratory functional impairment in sarcoidosis. Moreover, compared with the radiographic stages, HRCT findings appeared to be much more sensitive in depicting respiratory disability, especially abnormal gas exchange.

In a study in 2007, findings showed that HRCT reliably diagnosed MTB, and thus could help in predicting derangement of pulmonary function tests and gas exchange analysis in these patients. ${ }^{[6]}$ Sumikawa H, et al in 2006 found signs of interstitial fibrosis more frequently in IPF than in extrinsic aller- 
gic alveolitis (91.6\% versus 33.3\%). ${ }^{[7]}$ The presence of fibrosis with basal and peripheral distribution was characteristic of idiopathic pulmonary fibrosis, with good sensitivity and specificity $(75 \%)$, whereas in chronic extrinsic allergic alveolitis the areas of fibrosis often presented an irregular and heterogeneous distribution, in $91.6 \%$ of cases. However, $25 \%$ of extrinsic allergic alveolitis cases had a distribution mimicking idiopathic pulmonary fibrosis. The presence of areas with increased ground- glass opacity is more common in EAA than in IPF (66.6\% versus $33.3 \%) .{ }^{[6]}$ In our study also, the basal and subpleural distribution of honeycombing in IPF was well appreciated on HRCT. However appreciation of the same on conventional chest radiography was difficult.

Another study in 2005 found that the numbers of small nodular opacities in HRCT scans were significantly higher than those seen in radiography in all lung zones $(\mathrm{p}<0.01)$. HRCT was more sensitive than radiography in detecting small opacities of mid- out zones of the lung, but no statistical significance was found between the two methods in the detection of small opacities of lower zones of the lung. A statistically significant increase in the detectability of bulla, emphysema, pleural, mediastinal and hilar changes was observed $(p<0.05)$. HRCT might also be more sensitive than radiography in detecting lung parenchymal changes suggestive of silicosis. ${ }^{[8]}$ The findings in our study also revealed similar results with HRCT being able to detect nodular opacity in 19 of the 30 cases $(63 \%)$ while chest radiography could detect the same in only 11 of the 30 cases (37\%).

In 2008 Sun J et al reported that HRCT is more reproducible and accurate than radiography, as suggested by the higher agreement between readers and the better correlation with pulmonary function tests, irrespective of smoking and chronic bronchitis; however, these data do not support the hypothesis that HRCT is more sensitive than chest radiography in the early detection of silicosis. There was poor concordance between chest radiography and HRCT in the early stage of silicosis. ${ }^{[9]} \mathrm{We}$ could detect more reticular opacities, septal thickening and lymphadenopathy in silicosis by HRCT than conventional chest radiogram. However the difference was not statistically significant (probably due to the small number of cases).

Profusion of opacities on HRCT correlates with functional impairment. The presence of branching centrilobular structures may be helpful in early recognition of silicosis. ${ }^{[10]}$ High resolution computed tomography is a diagnostic method of choice in the evaluation of lung parenchyma. HRCT enables the evaluation of small interstitial changes, invisible on plain chest radiographs, and their assessment at the level of the lung lobule. Nodular thickening of the peribronchovascular interstitium and interlobular septa are typical in lymphangitic spread of carcinoma. Smooth peribronchovascular and septal thickenings are typical in sarcoidosis, and are only seen in some patients in the lymphangitic spread of carcinoma. In lymphangitis carcinomatosa lung architecture remains unchanged, which allows differentiation from sarcoidosis. ${ }^{[5]}$ In our study also, we were able to appreciate the different types of septal thickening evident in different diseases; although detailed statistical analysis in this regard was not performed.

Falaschi $\mathrm{F}$ et al did a study on silicosis whereby they found that HRCT abnormalities were identified in $70 \%$, pulmonary function abnormalities were present in only $41 \%$, and the plain chest radiograph was abnormal in only $24 \%$. The most common HRCT findings were: interstitial lung disease, bronchiectasis, mediastinal or axillary lymphadenopathy, and pleuropericardial abnormalities. ${ }^{[1]}$ The results of this study, the first to describe the HRCT findings in SLE, suggest that airways disease, lymphadenopathy, and interstitial lung disease are common thoracic manifestations of SLE, whereas pleural abnormalities are less common than previously suggested. HRCT evidence of airways disease and interstitial lung disease was frequently present despite an absence of symptoms, a normal chest radiograph, and normal pulmonary function testing.

A study on rheumatoid arthritis in 2007 showed that interstitial involvement was the most common finding on HRCT $(36 \%)$ and followed by air trapping (20\%). Bronchiectasis, pulmonary nodule, and pleural disease were seen in $16 \%$, $12 \%$, and $12 \%$ of patients, respectively. None of patients had emphysema and honeycomb pattern. There was no statistically significant correlation between HRCT findings and disease activity criteria. ${ }^{[12]}$ In our study in the identification of pleural disease, reticular opacities and ground glass haziness, HRCT could detect more number of cases.

In another study, HRCT in rheumatoid arthritis revealed air trapping in $69 \%$, bronchiectasis in $58 \%$, bronchial wall thickening in $52 \%$ and ground glass opacities (GGOs) in $35 \%$ of the patients. Pleural thickening and effusion were observed in $11 \%$. Chest radiogram was abnormal in only one patient revealing a single pulmonary nodule. ${ }^{[13]}$

The inference that we draw from this study is that HRCT is much more sensitive than conventional chest radiography in the assessment and diagnosis of patients with interstitial lung diseases. Hence, HRCT seems to be the investigation of choice for the evaluation of the parenchymal abnormalities in interstitial lung diseases and thereby make a more specific and accurate diagnosis of the particular disease.

\section{Conclusion}

The diagnosis of interstitial lung disease (ILD) is frequently delayed because clinical clues are neglected and respiratory symptoms are ascribed to more common pulmonary diagnoses such as chronic obstructive pulmonary disease (COPD) in the primary care setting. While ILD cases ultimately require 
referral to a pulmonologist, many cases can be diagnosed in the early stages with the help of HRCT. HRCT is able to detect abnormalities in patients when the clinical signs are minimal or even when the chest radiograph appears completely normal.

Chest radiography is a relatively insensitive modality of investigation for the diagnosis of ILDs. Ultimately all patients with clinical suspicion of ILDs should benefit from an HRCT scan of the thorax. High resolution computed tomography (HRCT) chest scans are essential to the diagnostic work-up since each ILD form is characterised by a specific pattern of abnormalities and a confident diagnosis can often be arrived at by HRCT alone or in correlation with the clinical symptoms. When HRCT findings are characteristic in appropriate clinical settings, HRCT may even obviate the need for a lung biopsy.

\section{References}

1. Neurohr C, Behr J. Diagnostik und Therapie interstitieller Lungenerkrankungen. Dtsch Med Wochenschr. 2009;134(11):524529. Available from: https://dx.doi.org/10.1055/s-00291208082

2. Mayeux R. Biomarkers: Potential Uses and Limitations. Neuro. 2004;1(2):182-188. Available from: https://dx.doi.org/ 10.1602/neurorx.1.2.182.

3. Bessis L, Callard P, Gotheil C, Biaggi A, Grenier P. Highresolution $\mathrm{CT}$ of parenchymal lung disease: precise correlation with histologic findings. Radiographics. 1992;12(1):45-58. Available from: https://dx.doi.org/10.1148/radiographics.12.1. 1734481.

4. King TE. Clinical Advances in the Diagnosis and Therapy of the Interstitial Lung Diseases. Am J Respir Crit Care Med. 2005;172(3):268-279. Available from: https://dx.doi.org/10. 1164/rccm.200503-483oe.

5. Bourke SJ. Interstitial lung disease: progress and problems. Postgrad Med J. 2006;82(970):494-499. Available from: https: //dx.doi.org/10.1136/pgmj.2006.046417.

6. Pipavath SN, Sharma SK, Sinha S, Mukhopadhyay S, Gulati MS. High resolution CT (HRCT) in miliary tuberculosis (MTB) of the lung: Correlation with pulmonary function tests\& gas exchange parameters in north Indian patients. Indian J Med Res. 2007;126(3):193-201.

7. Sumikawa H, Johkoh T, Ichikado K, Taniguchi H, Kondoh Y, Fujimoto K, et al. Usual Interstitial Pneumonia and Chronic Idiopathic Interstitial Pneumonia: Analysis of CT Appearance in 92 Patients. Radiology. 2006;241(1):258-266. Available from: https://dx.doi.org/10.1148/radiol.2411050928.

8. Sun $\mathrm{J}$, Weng $\mathrm{D}$, Jin $\mathrm{C}$, Yan $\mathrm{B}, \mathrm{Xu} \mathrm{G}$, Jin $\mathrm{B}$, et al. The Value of High Resolution Computed Tomography in the Diagnostics of Small Opacities and Complications of Silicosis in Mine Machinery Manufacturing Workers, Compared to Radiography. J Occup Health. 2008;50(5):400-405. Available from: https://dx.doi.org/10.1539/joh.18015.

9. Schurawitzki H, Stiglbauer R, Graninger W, Herold C, Pölzleitner D, Burghuber OC, et al. Interstitial lung disease in progressive systemic sclerosis: high-resolution CT versus radiography. Radiology. 1990;176(3):755-759. Available from: https://dx.doi.org/10.1148/radiology.176.3.2389033.

10. dos Santos Antao VC, Pinheiro GA, Terra-Filho M, Kavakama J, Müller NL. High-resolution CT in silicosis: correlation with radiographic findings and functional impairment. J Comput Assist Tomogr. 2005;29(3):350-356. Available from: https: //doi.org/10.1097/01.rct.0000160424.56261.bc.

11. Falaschi F, Battolla L, Paolicchi A, Paggiaro P, Talini D, Carrara M, et al. High-resolution computed tomography compared with the thoracic radiogram and respiratory function tests in assessing workers exposed to silica. Radiol Med. 1995;89(4):424-433.

12. Karazincir S, Akoğlu S, Güler H, Balci A, Babayiğit C, Eğilmez E. The evaluation of early pulmonary involvement with high resolution computerized tomography in asymptomatic and nonsmoker patients with rheumatoid arthritis. Tuberk Toraks. 2009;57(1):14-21.

13. Metafratzi ZM, Georgiadis AN, Ioannidou CV, Alamanos Y, Vassiliou MP, Zikou AK, et al. Pulmonary involvement in patients with early rheumatoid arthritis. Scand J Rheumatol. 2007;36(5):338-344. Available from: https://dx.doi.org/10 1080/03009740701393957.

Copyright: (C) the author(s), 2020. It is an open-access article distributed under the terms of the Creative Commons Attribution License (CC BY 4.0), which permits authors to retain ownership of the copyright for their content, and allow anyone to download, reuse, reprint, modify, distribute and/or copy the content as long as the original authors and source are cited.

How to cite this article: Madhu P, Santhosh D. Comparison between Conventional Radiography and High Resolution Computed Tomography in Interstitial Disease of Lungs. Asian J. Med. Radiol. Res. 2020;8(1):159-165.

DOI: dx.doi.org/10.47009/ajmrr.2020.8.1.29

Source of Support: Nil, Conflict of Interest: None declared. 